\title{
Efficient Algorithms for Optimal Video Transmission
}

\author{
Dexter Kozen \\ kozen@cs . cornell.edu
}

\author{
Yaron Minsky \\ yminsky@cs . cornell .edu \\ Computer Science Department \\ Upson Hall \\ Cornell University \\ Ithaca, New York 14853-7501
}

\author{
Brian Smith \\ bsmith@cs.cornell.edu
}

May 16, 1995

\begin{abstract}
This paper addresses the problem of sending an encoded video stream over a channel of limited bandwidth. When there is insufficient bandwidth available, some data must be dropped. For many video encodings, some data are more important that others, leading to a natural prioritization of the data. In this paper we give fast algorithms to determine a prioritization which optimizes the visual quality of the received data. By "optimized visual quality," we mean that the expected maximum interval of unplayable frames is minimized.

Our results are obtained in a model of encoded video data that is applicable to many encoding technologies. The highlight of the model is an interesting relationship between the play order and dependence order of frames. The property allows fast determination of optimal send orders by dynamic programming and is satisfied by all MPEG sequences.
\end{abstract}

\section{Introduction}

The transmission requirements for video data and ordinary data are quite different. Ordinary data need not be received in real time, but every bit must be correct. Video data, on the other hand, can tolerate some loss, but must be received, decoded, and played back in real time. Unfortunately, limitations on channel bandwidth may not allow all the data to get through for real-time playback. The question then arises: under the assumption that some data will be lost, which data should be sent, and in what order, so as to optimize the perceived quality of the received video stream?

For example, consider a video segment consisting of 120 frames, all the same size and independently de- codable. Suppose further that due to bandwidth constraints we know that only 90 frames will get through in the allotted time. The question is: which frames should be sent? Sending the frames in the order of play would leave a 30 -frame gap at the end, which at a playback rate of 30 frames/sec would be perceived by the viewer as a one-second discontinuity. A better choice would be to drop every fourth frame, because the gaps are small and evenly distributed.

The approach of dropping frames in the absence of sufficient bandwidth is known as temporal decimation. Determining which frames to drop is easy if all frames are the same size and there are no dependencies, as with the example above. However, the problem is significantly more complicated without these restrictions.

Such is the case with the MPEG encoding standard [4]. Dependencies between frames arise because frames that are very similar to neighboring frames are not represented in their entirety, but encoded succinctly as modifications to neighboring frames. This gives considerable savings in storage and transmission time, but has the disadvantage that to decode a frame, the receiving process must also have access to all the frames it depends on. When considering which frames to drop from a video stream, the sending process is confronted with the fact that some frames are more important than others. For example, if it drops a frame upon which many others depend, the effect on the final video might be disastrous. Another complicating factor is that the sizes of the encoded frames vary significantly due to dependencies and compression, in practice from $1 \mathrm{~K}$ to $12 \mathrm{~K}$ bytes. A third consideration is that over traditional networks, available bandwidth may be highly variable, depending on network characteristics such as link capacities and network load. 
These observations have lead many researchers to develop prioritized transmission schemes. For example, Priority Encoded Transmission (PET) [1] uses a variation on forward error correction where redundant data is introduced to compensate for data loss. Priority is encoded by associating high redundancy with more important frames. Cyclic-UDP [6] uses a retransmission scheme that allows for prioritized delivery. Reservation networks such as Tenet [3] allow users to reserve bandwidth in the network, with statistical guarantees on the transmission properties of the packets. Prioritized delivery can be obtained using several channels. For example, a 1.5 Mbit/sec channel could be divided into a $1.0 \mathrm{Mbit} / \mathrm{sec}$ channel that has no packet loss, and a $0.5 \mathrm{Mbit} / \mathrm{sec}$ channel that has $20 \%$ packet loss. One would then place high priority data on the first channel and low priority data on the second.

An open problem in all of these systems is to sensibly choose a prioritization order for MPEG video data. It is this problem we address in this paper.

Our solution assumes a transport layer that implements some priority-based transmission scheme, which, when provided with $n$ packets in priority order, will transmit the $k$ highest priority packets. We model $k$ by a random variable distributed in the set $\{0,1, \ldots, m\}$ according to some probability distribution $\mathbf{P r}$, where $m$ is the total number of packets. For instance, $\operatorname{Pr}(k=0)$ is the probability that no data will be received, while $\operatorname{Pr}(k=m)$ is the probability that all packets get through.

In this paper we give polynomial-time algorithms to determine optimal transmission orders. The transmission orders that we compute are optimal in the sense that they minimize the expected maximum interval of unplayable frames, under reasonable assumptions on the distribution of the channel bandwidth. A frame is unplayable if either it was not received, or it was received but cannot be decoded because it depends on a frame that was not received.

We give two algorithms for two different situations. The first assumes that we know the channel bandwidth exactly. This would be the situation for example with a reservation-based ATM network, where the network server provides a guaranteed bandwidth to the sending process for an interval of time. The algorithm calculates in time $O\left(m n^{3}\right)$ a table that can be stored with the video data that for each $k \leq m$ gives a set of frames of total weight at most $k$ that minimizes the maximum interval of unplayable frames.

The second algorithm does not assume known bandwidth, but assumes that the bandwidth is uniformly distributed in an interval with known endpoints. This assumption is realistic, since there exist techniques to measure the channel bandwidth at any instant of time quite reliably [6]. The algorithm computes in time $O\left(p m n^{3}\right)$ an optimal order to transmit the frames, where $p$ is the size of the interval. The order is optimal in the sense that it minimizes the expected maximum interval of unplayable frames.

We remark that the obvious greedy algorithm (in each step, choose the enabled frame that minimizes the maximum gap size of the remaining set of frames; continue until the cutoff $k$ is exceeded) is not optimal in either case.

All our work is carried out in the context of a simple combinatorial model of encoded video data that is applicable to most encoding technologies. The model consists of a set of frames, a partial order on the frames called the dependence order, a total order on the frames called the play order, and weight function giving the size of each frame. The highlight of the model is an interesting formal relationship between the dependence order and the play order, namely: the set of frames depending on any single frame is an interval in the play order. It may be difficult at first to see the significance of this condition, but it turns out to be exactly the technical property required to allow optimal send orders to be calculated efficiently. Fortuitously, this property is satisfied by all MPEGencoded video sequences. It leads to an independence property (Theorem 3) that allows a dynamic programming solution. We discuss the intuitive significance of this property more fully in $\S 2.2$.

As mentioned, the problem we solve in this paper is part of an approach to the general problem called temporal decimation, in which selected frames are dropped from the encoded sequence of frames. The missing frames are filled in by replaying the latest decoded frame or by interpolation [5]. As more frames are dropped, the image degrades by developing a jerkiness, depending on the sizes of the gaps between playable frames and the quality of the interpolation process. While temporal decimation is standard practice for some kinds of compressed video, it has generally not been used with MPEG streams because of the frame dependencies.

A more common approach, popular with the signal processing community, is spatial decimation. This takes the form of dropping the least significant coefficients of the Fourier or cosine transform of the image [2]. As more data are dropped, the image degrades by developing a halo around the edges of objects, called 
the corona effect. This approach is highly dependent on the coding method and data format, whereas the temporal approach is relatively independent of the particular coding method. We predict that a combination of the two approaches will produce the most effective results in practice.

\section{$2 \quad$ A Model}

We model an encoded video sequence by:

- a finite set $U$ of frames,

- a total order $\sqsubseteq$ on $U$ called the play order, intuitively the chronological order of the frames,

- a partial order $\preceq$ on $U$ called the dependence order, intuitively the order giving the dependence of one frame on another as described in the introduction. We write $u \preceq v$ to indicate that frame $v$ depends on $u$, i.e. cannot be decoded without knowledge of $u$; and

- a weight function $\omega: U \rightarrow \mathbb{N}$ giving the number of data units (nominally packets) comprising each frame.

We also postulate the following key condition describing the relationship between the two orders $\sqsubseteq$ and $\preceq$ :

Condition 1 For any frame $u$, the set of frames depending on u form an $\sqsubseteq$-interval. In other words,

$$
\forall u \exists x \exists z\{v \mid u \preceq v\}=\{y \mid x \sqsubseteq y \sqsubseteq z\} .
$$

Although in reality if $u \preceq v$ then $u$ is typically larger than $v$, we do not assume any formal relationship between $\preceq$ and $\omega$ in the model; nor do we take advantage of any special structure of MPEG other than Condition 1 .

Let $\leq$ be any partial order on $U$. We write $u<v$ if $u \leq v$ but not $v \leq u$. For $u \in U$ and $A \subseteq U$, define

$$
\begin{aligned}
& u \uparrow \leq=\{v \mid u \leq v\} \\
& A \uparrow \leq=\bigcup_{u \in A} u \uparrow \leq=\{v \mid \exists u \in A u \leq v\} .
\end{aligned}
$$

$u \downarrow_{\leq}$and $A \downarrow \leq$ are defined similarly. For $V \subseteq U$, a $\leq$ suffix of $V$ is a subset $X \subseteq V$ such that $X=V \cap X \uparrow \leq$, i.e. it is closed upward under $\leq$. A $\leq$-prefix of $V$ is a subset $X \subseteq V$ such that $X=V \cap X \downarrow_{\leq}$, i.e. it is closed downward under $\leq$. An $\leq$-interval of $V$ is an intersection of a suffix of $V$ and a prefix of $V$.

In this notation, Condition 1 says that for all frames $u, u \uparrow \preceq$ is an $\sqsubseteq$-interval.
For any set $A$, denote the cardinality of $A$ by $|A|$. For $A \subseteq U$, define $\omega(A)=\sum_{u \in A} \omega(u)$. A send order is a map $\pi:\{1,2, \ldots, \omega(U)\} \rightarrow U$ such that for any $u$, $\left|\pi^{-1}(u)\right|=\omega(u)$. Intuitively, at time $i$, we send the next packet from frame $\pi(i)$.

For a send order $\pi, u \in U, A \subseteq U$, and $0 \leq i \leq \omega(U)$, define

$$
\begin{aligned}
\operatorname{last}_{\pi}(u) & \stackrel{\text { def }}{=} \max \pi^{-1}(u) \\
\operatorname{last}_{\pi}(A) & \stackrel{\text { def }}{=} \max _{u \in A} \operatorname{last}_{\pi}(u) \\
\operatorname{received}_{\pi}(i) & \stackrel{\text { def }}{=}\left\{u \mid \operatorname{last}_{\pi}(u) \leq i\right\} \\
\operatorname{playable}_{\pi}(i) & \stackrel{\text { def }}{=}\left\{u \mid u \downarrow \preceq \subseteq \operatorname{received}_{\pi}(i)\right\} \\
& =\left\{u \mid \operatorname{last}_{\pi}(u \downarrow \underline{\jmath}) \leq i\right\} \\
\text { unplayable }_{\pi}(i) & \stackrel{\text { def }}{=} U-\operatorname{playable}_{\pi}(i) \\
\operatorname{maxint}_{(A)} & \stackrel{\text { def }}{=} \max \{|I| \mid I \text { is an } \sqsubseteq \text {-interval } \\
\operatorname{gap}_{\pi}(i) & \stackrel{\text { def }}{=} \quad \operatorname{maxint}\left(\operatorname{unplayable}{ }_{\pi}(i)\right) \\
\mathcal{E}\left(\operatorname{gap}_{\pi}(k)\right) & \stackrel{\text { def }}{=} \sum_{i=0} \operatorname{gap}_{\pi}(i) \mathbf{P r}(k=i)
\end{aligned}
$$

The number last ${ }_{\pi}(u)$ represents the time that the last packet of $u$ is sent under the send order $\pi$, and last $\pi(A)$ is the time that the last packet of some frame in $A$ is sent under the send order $\pi$. When $\pi$ is the send order and $i$ is the cutoff, the set $\operatorname{received}_{\pi}(i)$ is the set of frames received; the set playable $_{\pi}(i)$ is the set of playable frames (the set of frames $u$ such that $u$ and all frames on which $u$ depends are received); the set unplayable $e_{\pi}(i)$ is the set of unplayable frames; and the number $\operatorname{gap}_{\pi}(i)$ is the length of the longest unplayable interval. The quantity $\mathcal{E}\left(\operatorname{gap}_{\pi}(k)\right)$ is the expected length of the longest unplayable interval when $\pi$ is the send order. A send order $\pi$ is optimal if $\mathcal{E}\left(\operatorname{gap}_{\pi}(k)\right)$ is minimum.

The send order $\pi$ defines a total order on frames according to the time at which their last packets are sent:

$$
u \unlhd_{\pi} v \stackrel{\text { def }}{\Longleftrightarrow} \operatorname{last}_{\pi}(u) \leq \operatorname{last}_{\pi}(v)
$$

\subsection{Acceptable Send Orders}

Define a send order $\pi$ to be contiguous if for all $u \in U$, $\pi^{-1}(u)$ is an $\leq$-interval. In other words, all packets of one frame are sent together. Define a send order $\pi$ to be consistent with the dependence order $\preceq$ if

$$
u \preceq v \Rightarrow u \unlhd_{\pi} v
$$


i.e. $\unlhd_{\pi}$ is a total extension of $\preceq$. We say that a send order is acceptable if it is both contiguous and consistent with $\preceq$.

We show that without loss of generality we can restrict our attention to acceptable send orders. Intuitively, a frame is not considered received until its last packet is received; and it does not make sense to send a frame unless all frames on which it depends have already been sent, since it cannot be decoded without them anyway.

Theorem 2 For any send order $\pi$, there is an acceptable send order $\pi^{\prime}$ such that

$$
\mathcal{E}\left(\operatorname{gap}_{\pi^{\prime}}(k)\right) \leq \mathcal{E}\left(\operatorname{gap}_{\pi}(k)\right)
$$

Proof. First we show that for any send order $\pi$, there is a contiguous send order $\pi^{\prime}$ such that (9) holds. Let $\pi^{\prime}$ be the unique contiguous send order such that $\unlhd_{\pi}=\unlhd_{\pi^{\prime}}$. The send order $\pi^{\prime}$ can be computed from $\pi$ by rearranging the packets so that all packets belonging to the last frame $\pi(\omega(U))$ appear contiguously at the end of the sequence, and otherwise keeping the order of the packets intact; then repeating with the next-to-last frame, etc. The resulting send order $\pi^{\prime}$ satisfies

$$
\operatorname{last}_{\pi^{\prime}}(u) \leq \operatorname{last}_{\pi}(u)
$$

for all frames $u$. Then (9) follows easily from the definitions (1) $-(8)$.

Now let $\pi$ be any contiguous send order. We show that there is an acceptable send order $\pi^{\prime}$ such that (9) holds. We show how to rearrange the frames such that contiguity is maintained, consistency with $\preceq$ is improved, and $\mathcal{E}\left(\operatorname{gap}_{\pi}(k)\right)$ does not increase.

Let $u, v$ be such that $u \unlhd_{\pi} v$ but $v \prec u$ and the $\triangleleft_{\pi^{-}}$ interval between $u$ and $v$ is of minimal size. Then $u \npreceq w$ for any $u \triangleleft_{\pi} w \unlhd_{\pi} v$. Let $\pi^{\prime}$ be the send order obtained by moving $u$ immediately after $v$. We have $\operatorname{last}_{\pi^{\prime}}(w) \leq \operatorname{last}_{\pi}(w)$ for all frames $w$ except possibly $u$, and $\operatorname{last}_{\pi^{\prime}}(u \downarrow \preceq)=\operatorname{last}_{\pi}\left(u \downarrow_{\preceq}\right)$, since $v \in u \downarrow \preceq$. Thus last $\pi^{\prime}\left(w \downarrow_{\preceq}\right) \leq \operatorname{last}_{\pi}\left(w \downarrow_{\preceq}\right)$ for all $w$. Again, $(\overline{9})$ follows easily from the definitions (1) $-(8)$.

Note that an acceptable send order $\pi$ is uniquely determined by its induced total order $\unlhd_{\pi}$ on $U$; for this reason we occasionally omit the $\pi$ and speak of send orders $\unlhd$.

\subsection{Independence of Gaps}

Let $\leq$ be a partial order on $U$. Two sets $V, W \subseteq U$ are said to be $\leq$-independent if no pair of elements $v \in V$ and $w \in W$ are $\leq$-comparable; i.e., if for all $v \in V$ and $w \in W$, neither $v \leq w$ nor $w \leq v$. A family of subsets of $U$ is said to be pairwise $\leq-$ independent if all pairs of sets in the family are $\leq$-independent.

Let $V \subseteq U$. We say that a set $A$ is enabled in $V$ if $A$ is a $\preceq$-prefix of $V$. A set is enabled if it is enabled in $U$. A single element $u$ is enabled in $V$ if $\{u\}$ is enabled in $V$, i.e. if $u$ is a $\preceq$-minimal element of $V$. The set of enabled elements of $V$ is denoted enabled( $V)$. A gap of an enabled set $A$ is a maximal $\sqsubseteq$-interval of $U-A$.

The following independence property is the crucial observation that allows optimal send orders to be computed efficiently.

Theorem 3 The set of gaps of any enabled set are pairwise $\preceq$-independent.

Proof. It suffices to show that if $A$ is enabled, $w \in A$, $u, v \in U-A$, and $u \sqsubset w \sqsubset v$, then $u$ and $v$ are $\preceq$-incomparable. In other words, if $A$ is enabled and $\bar{w} \in A$, then $w \downarrow \downarrow \cap(U-A)$ and $w \uparrow \sqsubseteq \cap(U-A)$ are $\preceq$-independent.

Suppose $u \preceq v$. By Condition 1 , the set $u \uparrow \preceq$ is an $\sqsubseteq$-interval containing $u$ and $v$, therefore also contains $w$. But then

$$
u \in w \downarrow \preceq \subseteq A \downarrow \preceq=A,
$$

contradicting the assumption $u \notin A$. The assumption $v \preceq u$ leads similarly to a contradiction.

Intuitively, if we have decided to send a set of frames $A$, and $w \in A$, then no remaining frame occurring before $w$ in the play order sent in the future can cause a frame occurring after $w$ in the play order to become enabled, or vice versa. This will allow us to process the $\sqsubseteq$-intervals to the left and right of $w$ independently to get optimal send orders on those subsets, then merge them to get an optimal send order on the union.

\subsection{Other Basic Properties}

We conclude this section with some other useful properties.

Lemma 4 The set $A$ is enabled in $V$, i.e. $A$ is $a \preceq$ prefix of $V$, iff there is an acceptable send order $\pi$ with respect to which $A$ is a $\unlhd_{\pi}$-prefix of $V$.

Proof. Suppose $A$ is a $\unlhd_{\pi}$-prefix of $V$. Then $A=$ $A \downarrow_{\unlhd_{\pi}} \cap V$, hence $A \downarrow_{\preceq} \cap V \subseteq A \downarrow_{\unlhd_{\pi}} \cap V=A$, so $A$ is a $\preceq$-prefix of $V$. 
Conversely, suppose $A$ is a $\preceq$-prefix of $V$. Consider the relation $\preceq \cup(A \times(V-A))$. This is a partial order extending $\preceq$. Any contiguous total order $\unlhd$ extending this order is acceptable because it includes $\preceq$, and $A$ is a $\unlhd$-prefix of $V$ because $\unlhd$ includes $A \times(V-A)$.

Lemma 5 Let $I \subseteq U$. The following statements are equivalent:

(i) I is both an 巨-interval and a $\preceq$-suffix of $U$.

(ii) I is a gap of some enabled set.

Proof. Suppose (i). Since $I$ is a $\preceq$-suffix of $U$, its complement $U-I$ is a $\preceq$-prefix of $U$, therefore enabled, and $I$ is a gap of $U-I$.

The converse follows directly from Theorem 3 .

Intuitively, an -interval $I$ is a $\preceq$-suffix iff there is a enabled set of frames containing the exterior endpoints of $I$ but disjoint from $I$. The $\sqsubseteq$-interval $I$ could be the $\sqsubseteq$-interval between two frames that have already been sent (or everything to the left or right of a frame that has already been sent), but nothing in $I$ has been sent yet.

Lemma 6 Any nonempty gap of an enabled set $A$ contains at least one element enabled in $U-A$.

Proof. Let $I$ be a nonempty gap of an enabled set $A$. By Theorem 3, any $\preceq$-minimal element of $I$ is enabled in $U-A$.

Lemma 7 Let $A$ be enabled, $I$ a gap of $A$, and $B \subseteq$ I. Then $B$ is enabled in $I$ if and only if it is enabled in $U-A$.

Proof. If $B$ is enabled in $U-A$, then it is enabled in $I$, since $I \subseteq U-A$. Conversely, if $B$ is enabled in $U-A$, then $I \cap B \downarrow \preceq=B$; and by Theorem $3, B \downarrow \preceq \subseteq A \cup I$. Thus

$$
\begin{aligned}
B & \downarrow \preceq \cap(U-A) \\
& =B \downarrow \preceq \cap((U-A-I) \cup I) \\
& =(B \downarrow \preceq \cap(U-A-I)) \cup(B \downarrow \preceq \cap I) \\
& =B .
\end{aligned}
$$

Lemma 8 Let $A$ be enabled, $I$ a gap of $A$, and $B$ enabled in $I$. Then $A \cup B$ is enabled.
Proof. Since $A$ is enabled, by Lemma 7 we have

$$
\begin{aligned}
A \cup B \downarrow \preceq & =A \downarrow \preceq \cup B \downarrow \preceq \\
& =A \cup(B \downarrow \preceq \cap(U-A)) \\
& =A \cup B .
\end{aligned}
$$

\section{Algorithms for Known Cutoff}

Algorithm 9 below is a recursive algorithm for computing an enabled set $A$ minimizing maxint $(A)$ subject to $\omega(A) \leq k$ for known cutoff $k$. The order of transmission does not matter. The algorithm actually computes a table of such sets for all values of $k$ by dynamic programming. This corresponds to the case where the probability distribution is a point mass, so probabilistic arguments are not necessary.

The algorithm is called by $\operatorname{GAP}(I)$, where $I$ is a gap of some enabled set (which by Lemma 5 is any $\preceq-$ upward-closed $\sqsubseteq$-interval). It returns a list of subsets $B(d) \subseteq I$ and a list of numbers $C(d)$, one for each $0 \leq d \leq \omega(I)$, such that

(i) $B(d)$ is enabled in $I$

(ii) $\omega(B(d)) \leq d$

(iii) $C(d)=\operatorname{maxint}(I-B(d))$

(iv) $C(d)$ is minimum among all subsets of $I$ satisfying (i) and (ii).

The algorithm is called at the top level by $\operatorname{GAP}(U)$.

\section{Algorithm 9 GAP $(I)$ :}

If $I=\varnothing$, return lists with one element $B(0)=\varnothing$ and $C(0)=0$.

Otherwise, for each $u \in \operatorname{enabled}(I)$, set

$$
\begin{aligned}
& I_{\sqsubset u}=I \cap\{v \mid v \sqsubset u\} \\
& I_{\sqsupset u}=I \cap\{v \mid v \sqsupset u\} .
\end{aligned}
$$

By Lemma $8, I_{\sqsubset u}$ and $I_{\sqsupset u}$ are $\preceq$-suffixes of $U$. Recursively call $\operatorname{GAP}\left(I_{\sqsubset u}\right)$ and $\operatorname{GAP}\left(I_{\sqsupset u}\right)$. This gives $B_{\sqsubset u}$, $C_{\sqsubset u}, B_{\sqsupset u}$, and $C_{\sqsupset u}$. Create the lists $B_{I, u}$ and $C_{I, u}$ that give the optimal sets to send and their max gap sizes given that $u$ is one of the frames sent, as follows. For each $d<\omega(u)$, set

$$
\begin{aligned}
& B_{I, u}(d)=\varnothing \\
& C_{I, u}(d)=|I| .
\end{aligned}
$$


For each $d$ such that $\omega(u) \leq d \leq \omega(I)$, set

$$
\begin{aligned}
& B_{I, u}(d)=B_{\sqsubset u}(i) \cup\{u\} \cup B_{\sqsupset u}(j) \\
& C_{I, u}(d)=\max C_{\sqsubset u}(i), C_{\sqsupset u}(j)
\end{aligned}
$$

where $i, j$ are such that $0 \leq i+j+\omega(u) \leq d$ and

$$
\max C_{\sqsubset u}(i), C_{\sqsupset u}(j)
$$

is minimum. The set $B_{I, u}(d)$ is enabled in $I$ by Lemma 8. Using the fact that $C_{\sqsubset u}(i)$ and $C_{\sqsupset u}(i)$ are monotonically nonincreasing in $i$, one can scan the lists $C_{\sqsubset u}$ and $C_{\sqsupset u}$ linearly to determine $B_{I, u}$ and $C_{I, u}$ in time $O(\omega(I))$.

Now for each $d, 0 \leq d \leq \omega(I)$, set $B_{I}(d)=B_{I, u}(d)$ and $C_{I}(d)=C_{I, u}(d)$, where $C_{I, u}(d)$ is minimum over all enabled $u \in I$. This requires linear time in the sizes of the lists.

This algorithm can be implemented in a dynamic programming style by computing $\operatorname{GAP}(I)$ for all $\preceq$ upward closed $\sqsubseteq$-intervals $I$ of length 1 , then the same for all such $I$ of length 2, and so on. Alternatively, the algorithm can be implemented in a recursive style as given above, with intermediate results cached so that subsequent calls with the same parameters take constant time.

There are at most $O\left(n^{2}\right)$ intervals on which the procedure can be called. A call on interval $I$ takes time $O(\omega(I) \cdot|I|)$ exclusive of recursive subcalls, giving a total time bound of $O\left(\omega(U) \cdot n^{3}\right)$.

\section{Algorithms for Unknown Cutoff}

In this section, we do not assume that the cutoff $k$ is known, but is uniformly distributed on some interval $[a, b]$. We give the algorithm here for $a=0$ and $b=\omega(U)$, then discuss in $\S 4.2$ how to modify it for an arbitrary interval at a cost of an extra linear factor in complexity. In fact, we can give a polynomial-time algorithm for any step function with a constant number of steps, although this is not very interesting because the dependence is exponential in the number of steps of the step function.

Assume then that $k$ is uniformly distributed in the interval $[0, \omega(U)]$. Thus

$$
\operatorname{Pr}(k=i)=\frac{1}{\omega(U)+1} .
$$

In this case, (8) simplifies to

$$
\mathcal{E}\left(\operatorname{gap}_{\pi}(k)\right)=\frac{1}{\omega(U)+1} \sum_{u \in U} \operatorname{maxint}\left(u \uparrow_{\unlhd}\right) \omega(u) .
$$

Since all we care about is minimization, we can drop the normalization term $1 /(\omega(U)+1)$ and just minimize the sum over all choices of send order $\unlhd$.

\subsection{Greedy Merge}

The following result allows us to treat $\preceq$-independent sets separately, then combine the results to give an optimal solution for the union of the two sets.

Let $V, W$ be distinct gaps of some enabled set $A$. By Theorem $3, V$ and $W$ are $\preceq$-independent $\preceq$-suffixes of $U$, and any $\sqsubseteq$-interval intersecting both $V$ and $W$ also intersects $A$.

We say that a send order $\unlhd$ on $V \cup W$ is a greedy merge of its restrictions to $V$ and $W$ if for all $u \in V \cup W$,

$$
\begin{aligned}
u \in V \Rightarrow & \operatorname{maxint}\left(u \uparrow_{\unlhd} \cap V\right) \\
& \geq \operatorname{maxint}\left(u \uparrow_{\unlhd} \cap W\right) \\
u \in W \Rightarrow & \operatorname{maxint}\left(u \uparrow_{\unlhd} \cap V\right) \\
& \leq \operatorname{maxint}\left(u \uparrow_{\unlhd} \cap W\right)
\end{aligned}
$$

A greedy merge of send orders on $V$ and $W$ can be computed by taking in each step either the least remaining element $v \in V$ or the least remaining element $w \in W$, depending on which of maxint $\left(v \uparrow_{\unlhd}\right)$ and maxint $\left(w \uparrow_{\unlhd}\right)$ is larger.

Lemma 10 Let $S$ be an arbitrary subset of $V \cup W$. Then

$$
\operatorname{maxint}(S)=\max \left\{\begin{array}{l}
\operatorname{maxint}(S \cap V) \\
\operatorname{maxint}(S \cap W)
\end{array}\right.
$$

In particular, for any element $u \in V \cup W$ and send order $\unlhd$ on $U$,

$$
\operatorname{maxint}\left(u \uparrow_{\unlhd}\right)=\max \left\{\begin{array}{l}
\operatorname{maxint}\left(u \uparrow_{\unlhd} \cap V\right) \\
\operatorname{maxint}\left(u \uparrow_{\unlhd} \cap W\right) .
\end{array}\right.
$$

Proof. Any interval of $V$ or $W$ is also an interval of $S$. Conversely, because of the restrictions on $V$ and $W$, any interval of $S$ is either an interval of $V$ or an interval of $W$.

Lemma 11 Suppose the send order $\unlhd$ on $V \cup W$ is the greedy merge of its restriction to $V$ and $W$, respectively. Then for all $u \in V \cup W$,

$$
\begin{aligned}
& \operatorname{maxint}\left(u \uparrow_{\unlhd}\right) \\
& = \begin{cases}\operatorname{maxint}\left(u \uparrow_{\unlhd} \cap V\right), & \text { if } u \in V, \\
\operatorname{maxint}\left(u \uparrow_{\unlhd} \cap W\right), & \text { if } u \in W .\end{cases}
\end{aligned}
$$

Proof. This follows immediately from (10), (11), and (12). 
Lemma 12 Let $\unlhd$ be any send order on $V \cup W$. Then

$$
\begin{aligned}
& \sum_{u \in V \cup W} \operatorname{maxint}\left(u \uparrow_{\unlhd}\right) \cdot \omega(u) \\
& \geq \sum_{u \in V} \operatorname{maxint}\left(u \uparrow_{\unlhd} \cap V\right) \cdot \omega(u) \\
& \quad+\sum_{u \in W} \operatorname{maxint}\left(u \uparrow_{\unlhd} \cap W\right) \cdot \omega(u),
\end{aligned}
$$

with equality holding when $\unlhd$ is the greedy merge of its restrictions to $V$ and $W$.

Proof.

$$
\begin{aligned}
& \sum_{u \in V \cup W} \operatorname{maxint}\left(u \uparrow_{\unlhd}\right) \cdot \omega(u) \\
& =\sum_{u \in V} \operatorname{maxint}\left(u \uparrow_{\unlhd}\right) \cdot \omega(u) \\
& \quad+\sum_{u \in W} \operatorname{maxint}\left(u \uparrow_{\unlhd}\right) \cdot \omega(u) \\
& \geq \sum_{u \in V} \operatorname{maxint}\left(u \uparrow_{\unlhd} \cap V\right) \cdot \omega(u) \\
& \quad+\sum_{u \in W} \operatorname{maxint}\left(u \uparrow_{\unlhd} \cap W\right) \cdot \omega(u),
\end{aligned}
$$

by (12) and the fact that $V$ and $W$ are disjoint. If $\unlhd$ is the greedy merge of its restrictions to $V$ and $W$, then equality holds by Lemma 11 .

Theorem 13 Any greedy merge of optimal send orders on $V$ and $W$ is an optimal send order on $V \cup W$.

Proof. Any merge of send orders on $V$ and $W$ is a send order on $V \cup W$, since $V$ and $W$ are $\preceq$-independent. That the merge of optimal send orders on $V$ and $W$ results in an optimal send order on $V \cup W$ is a straightforward consequence of Lemma 12.

This implies that the optimal acceptable send order for a gap $I$ of an enabled set is of the form $u$ followed by a greedy merge of optimal send orders on $I_{\sqsubset u}$ and $I_{\sqsupset u}$ for some $u$ enabled in $I$. This gives rise to the following recursive algorithm for computing a send order $\pi(I)$ for a given interval $I$ minimizing

$$
C(I) \stackrel{\text { def }}{=} \sum_{u \in I} \operatorname{maxint}\left(u \uparrow_{\unlhd} \cap I\right) \omega(u) .
$$

For each enabled element $u$ of $I$, separate the interval into $I_{\sqsubset u}$ and $I_{\sqsupset u}$ and recursively compute $\pi\left(I_{\sqsubset u}\right)$, $C\left(I_{\sqsubset u}\right), \pi\left(I_{\sqsupset u}\right)$, and $C\left(I_{\sqsupset u}\right)$. Set

$$
C(I)=\min _{u \in \operatorname{enabled}(I)}|I| \cdot \omega(u)+C\left(I_{\sqsubset u}\right)+C\left(I_{\sqsupset} u\right) .
$$

For the $u \in$ enabled $(I)$ giving the minimum, greedily merge the optimal send orders $\pi\left(I_{\sqsubset u}\right)$ and $\pi\left(I_{\sqsupset} u\right)$ and put $u$ in front to obtain an optimal send order on $I$.

As in Algorithm 9, the algorithm can be implemented either in a bottom-up dynamic programming style or in a recursive style in which intermediate results are cached. There are $O\left(n^{2}\right)$ possible intervals on which the algorithm can be called, and a call on interval $I$ takes time $O(|I|)$ exclusive of recursive subcalls. This gives a total time bound of $O\left(n^{3}\right)$.

\subsection{Interval Distributions}

A straightforward modification of the algorithm works for an arbitrary interval distribution $(a, b]$. (We use left-semiopen intervals for technical convenience; this is without loss of generality, since a send order is optimal with respect to $(0, b]$ iff it is optimal with respect to $[0, b]$.) We compute recursively, for each gap of an enabled set, a table giving the optimal send order for each interval distribution $\left(a^{\prime}, b^{\prime}\right]$ on that gap, for all $a^{\prime} \leq a$ and $b^{\prime}-a^{\prime} \leq b-a$.

Optimal send orders on independent gaps are greedily merged as above, with the following modification. Suppose we wish to merge two send orders $\pi_{1}:\left(0, \omega\left(U_{1}\right)\right] \rightarrow U_{1}$ and $\pi_{2}:\left(0, \omega\left(U_{2}\right)\right] \rightarrow U_{2}$ on independent gaps $U_{1}$ and $U_{2}$, optimal with respect to interval distributions $\left(a_{1}, b_{1}\right]$ and $\left(a_{2}, b_{2}\right]$, respectively. We partition $\left(0, \omega\left(U_{1}\right)\right]$ into intervals $A_{1}=\left(0, a_{1}\right]$, $B_{1}=\left(a_{1}, b_{1}\right]$, and $C_{1}=\left(b_{1}, \omega\left(U_{1}\right)\right]$, and partition $\left(0, \omega\left(U_{2}\right)\right]$ into intervals $A_{2}=\left(0, a_{2}\right], B_{2}=\left(a_{2}, b_{2}\right]$, and $C_{2}=\left(b_{2}, \omega\left(U_{2}\right)\right]$. We greedily merge $\pi_{1} \mid A_{1}$ and $\pi_{2}\left\lceil A_{2}\right.$, then $\pi_{1} \uparrow B_{1}$ and $\pi_{2}\left\lceil B_{2}\right.$, then $\pi_{1} \mid C_{1}$ and $\pi_{2} \mid C_{2}$, then concatenate the three resulting send orders. This gives a send order $\pi$ : $\left(0, \omega\left(U_{1} \cup U_{2}\right)\right] \rightarrow U_{1} \cup U_{2}$ that is a candidate for the optimal send order with respect to the interval distribution $\left(a_{1}+a_{2}, b_{1}+b_{2}\right]$. The merge that gives the best result for each interval distribution is retained, and all others are discarded. The proof of correctness is a straightforward but notationally cumbersome modification of the proof of Theorem 13, and an analysis similar to that of the previous section gives a time bound of $O\left(p m n^{3}\right)$, where $p=b-a$ is the size of the original interval.

\section{Practical Considerations}

A version of the algorithm of section $\S 3$ has been implemented and tested on standard MPEG benchmarks with reasonable visual results.

In practice, we process a video stream in chunks of a few seconds. In actual transmissions, this would 
give a few seconds of latency while a chunk of the received data is being reassembled for decoding and playback. This approach is thus less useful for live teleconferencing than for transmitting canned video, where latency is not a factor.

To our chagrin, we discovered from our initial implementation that the basic algorithm as described $\S 3$ does not utilize bandwidth fully. That is, it does not necessarily fill in gaps smaller than the maximum gap, even if residual bandwidth is available, since according to the measure of minimizing the maximum gap, it thinks both solutions are equally good. In practice, we have fixed this by modifying the algorithm to choose the solution that minimizes the number of maximum gaps among all solutions that minimize the maximum gap. We are also experimenting with choosing the solution that maximizes the minimum interval of playable frames among all solutions that minimize the maximum gap (interval of unplayable frames), or some lexicographic combination of these conditions. All of these conditions can be accommodated easily by our algorithm with at most a small constant factor increase in time and space.

Our algorithm is least successful in "sweeping pans", where the same size gap produces a much more acute visual discontinuity. This is not so much a problem with our algorithm as with the metric of minimizing the maximum gap. If the sweeping pans could be identified by automatic scene analysis or some other method, then they could be weighted so as to avoid dropping frames in those areas. Our algorithm could easily be modified to use the weighted gap sizes. We leave this as a topic for future investigation.

Finally, we would like to comment on the applicability of our algorithm to decode-bound applications in which data is not being transmitted over a channel, but the time to decode is the bottleneck. For such applications, one can reinterpret the weight $\omega(u)$ as the time to decode the frame $u$ instead of its size, and our algorithm applies verbatim.

\section{Acknowledgements}

We thank Dan Huttenlocher, Daniel Scharstein, Prasoon Tiwari, and Ramin Zabih for valuable comments. The support of the National Science Foundation under grants CCR-9317320 and CDA-9121783 and the U.S. Army Research Office through the ACSyAM branch of the Mathematical Sciences Institute of Cornell University under contract DAAL03-91-C-0027 is gratefully acknowledged.

\section{References}

[1] A. Albanese, J. Blomer, J. Edmonds, M.Luby, AND M. Sudah, Priority encoding transmission, Symposium on Foundations of Computer Science, (1994), pp. 604-612.

[2] A. Eleftheridas, S. Pejhan, and D. Anastassiou, Algorithms and performance evaluation of the XPhone multimedia communication system, in Proceedings of ACM Multimedia 93, New York, 1993, Association for Computing Machinery Press, pp. 311-320. (Anaheim, CA, August 1-6, 1993).

[3] D. Ferrari, A. BanerJea, AND H. Zhang, Network support for multimedia-a discussion of the Tenet approach, Computer Networks and ISDN Systems, 26 (1994), pp. 1267-1280.

[4] D. L. GALL, MPEG: a video compression standard for multimedia applications, Communications of the ACM, 34 (1991), pp. 46-58.

[5] D. SCHARSTEIN, Synthesizing new views from stereo data, in IEEE Workshop on Representations of Visual Scenes (in conjunction with ICCV'95), 1995. Submitted.

[6] B. C. SMITH, Implementation Techniques for Continuous Media Systems and Applications, $\mathrm{PhD}$ thesis, University of California at Berkeley, 1994. 